\title{
Effect of Perioperative Coagulation Monitoring by Sonoclot on Short-Term Thrombosis in Pediatric Liver Transplantation
}

\section{Ying Sun}

Nankai University

Min Zhu

Nankai University

Feng Liu

Nankai University

Lili Jia

Nankai univercity,Tianjin,China

Mingwei Sheng

Nankai Univercity,Tianjin

Sudeshnna Thapa

Tianjin medical university

Wenli Yu ( $\sim$ yzxwenliyu@163.com )

Tianjin First Central Hospital https://orcid.org/0000-0002-6374-1944

\section{Research}

Keywords: Liver transplantation, Coagulation, Sonoclot, Thrombosis

Posted Date: December 2nd, 2020

DOl: https://doi.org/10.21203/rs.3.rs-117479/v1

License: (c) (1) This work is licensed under a Creative Commons Attribution 4.0 International License. Read Full License 


\section{Abstract}

Background Sonoclot devices are used to monitor coagulation function in transplant patients. However, the appropriate value of Sonoclot parameters for reducing postoperative thrombosis in pediatric liver transplantation is not clear. Therefore, the aim of the study was to investigate the effect of coagulation monitoring by Sonoclot devices on early postoperative thrombosis after liver transplantation in children.

Method The clinical data of 200 children with elective liver transplantation were collected. The effects of coagulation function monitoring by Sonoclot devices $1 \mathrm{~h}$ after ischemia-reperfusion on early thrombosis were analyzed. Receiver operating characteristic (ROC) analysis was used to obtain the sensitivity and cutoff value of Sonoclot parameters for the diagnosis of thrombosis.

Results A total of $38(19 \%)$ patients had thrombosis complications. 23 cases (11.5\%) had postoperative hepatic artery thrombosis and 15 cases $(7.5 \%)$ had portal vein and hepatic vein thrombosis. Sonoclot coagulation parameters included the following: for the thrombosis group \& non-thrombosis group, whole blood activated clotting time (ACT): $178.5 \pm 33.2 \& 280.4 \pm 58.6(p<0.05)$, clotting rate $(\mathrm{CR}): 12.4 \pm 5.4 \&$ $11.5 \pm 5.7(p>0.05)$, and platelet function (PF): $3.0 \pm 1.4 \& 2.6 \pm 1.1(p>0.05)$. Univariate and multivariate analyses showed that ACT was an independent risk factor for postoperative thrombosis. ROC analysis found that the sensitivity of ACT for the diagnosis of postoperative thrombosis was $94.7 \%$, and the ACT cutoff value to predict early postoperative thrombosis was $228.5 \mathrm{~s}$.

Conclusion ACT after ischemia-reperfusion was a risk factor for early postoperative thrombosis. It is recommended that ACT be maintained above $228.5 \mathrm{~s}$ to reduce postoperative thrombosis.

\section{Trial registration}

Approval was obtained from the Ethics Committee of Tianjin First Center Hospital in China (Approval Number: 2016N0039KY), and written informed consent was obtained from eligible guardians. This study has been registered in clinical trial (ClinicalTrials.gov ID: ChiCTR1900026125). All processes were ethical.

\section{Background}

Pediatric liver transplantation is the preferred treatment for children suffering from end-stage liver disease $^{[1]}$. The postoperative short- and long-term survival rate has continuously improved, and the survival rate at 1,5 , and 10 years after surgery is as high as $95 \%, 80 \%$, and $60 \%$, respectively ${ }^{[2,3]}$. It is undeniable that there are still some problems after liver transplantation. Some large traumas and irritations, such as hepatic failure, long operation time, massive hemorrhage, blood transfusion, and perioperative ischemia and reperfusion, could cause coagulation disorders, which not only cause major intraoperative and postoperative blood loss, arrhythmia, thrombosis and other vascular complications but also affect postoperative graft function recovery and overall quality of life. Unlike adults, for children, postoperative thrombosis is the most serious and common complication ${ }^{[4]}$. Blood flow signals that are too fast or too slow may have a trend of developing into thrombosis, especially at the anastomotic site. 
There are many factors that influence thrombosis, among which anastomotic stenosis and hypercoagulability are important factors ${ }^{[5]}$. Therefore, except for surgical technique problem, perioperative coagulation monitoring is frequently performed during liver transplantation in children to guide pro- and anticoagulant therapies.

Sonoclot analyzer is a point-of-care coagulation monitoring system that assesses the viscoelastic properties of whole blood ${ }^{[6]}$. It is beneficial for blood to be analyzed at the bedside, and Sonoclot analyzers allow a faster turnaround time. In addition, Sonoclot analyzers assess the whole blood coagulation status and provide useful information on platelet function ${ }^{[7]}$. Sonoclot devices are used in situations of abnormal coagulation, such as cardiac and liver transplant surgery ${ }^{[8,9]}$. However, the use of a Sonoclot in pediatric liver transplantation, which involves complex coagulopathy, has not been well elucidated. Therefore, in this study, a Sonoclot analyzer was used to determine changes in coagulation function after ischemia-reperfusion and to explore the optimal coagulation values for reducing postoperative thrombosis.

\section{Methods}

\section{Patients}

The study was conducted at a transplant center (Tianjin First Central Hospital). After obtaining institutional ethics committee approval in our hospital, 200 patients aged between 6 months and 2 years who underwent selected liver transplantation for biliary atresia disease were enrolled in this observational study. The exclusion criteria were as follows: children with congenital heart disease, central nervous system disease, or retransplantation; patients treated with heparin drugs during surgery; and patients with severe arrhythmia, major bleeding and other complications during the operation. All the living donors were family members (fathers or mothers).

General anesthesia was performed on all patients with endotracheal intubation. Combined intravenous and inhalational (sevoflurane) anesthetics, muscle relaxants, and opioids were implemented in all patients to maintain intraoperative anesthesia. Traditional monitoring methods were used to monitor patients' vital signs during surgery; the invasive monitors included an intraarterial catheter and central venous catheter. All data were obtained from the liver transplant and anesthesia records, and the procedures were performed by the same group of surgeons.

\section{Blood Coagulation Monitoring}

According to a previous study, Sonoclot devices (Sienco, Inc., Arvada, CO, USA) can be used to monitor coagulation ${ }^{[9]}$. Additionally, in the clinic, coagulation function was routinely monitored in our department with Sonoclot devices during perioperative liver transplantation, especially after reperfusion. It was clarified that coagulation function disorder was exacerbated as the operation progresses. Activated 
partial thromboplastin time (APTT) was prolonged, platelet count (PLT) and fibrinogen (Fib) were

decreased and rebounded peak in the initial phase of the new-liver stage ${ }^{[10]}$. We found that from $1 \mathrm{~h}$ after reperfusion until the end of surgery, the coagulation function tended to be stable if there was no other intervention. Therefore, the Sonoclot coagulation monitoring data at $1 \mathrm{~h}$ after ischemia-reperfusion were collected, including whole blood activated clotting time (ACT), clotting rate (CR), and platelet function (PF). All data were stored on the Sonoclot machine.

\section{Clinical Data Collection}

We collected the following preoperative, intraoperative and postoperative data. The following preoperative variables were included: patient characteristics, pediatric end-stage liver disease (PELD) scores, serum bilirubin, serum albumin, aspartate aminotransferase (AST), alanine aminotransferase (ALT), and the international normalized ratio (INR). Intraoperative data were the graft cold ischemia time, anhepatic time, urine output, bleeding volume, blood products and the duration of surgery. Postoperative data included the highest AST, ALT and total bilirubin levels during the first week after the operation, the intensive care unit (ICU) stay time, and other complications such as mortality, secondary transplant, and abdominal laparotomy one month after transplantation. After discharge from the hospital, the children were followed up regularly through the hospital follow-up center to monitor their condition.

\section{Thrombosis Diagnostic Criteria}

We followed up and observed the occurrence of thrombosis within one week after surgery. The diagnostic criteria for thrombosis were as follows ${ }^{[11]}$ : 1. Postoperative transaminase and bilirubin levels were important indicators for early diagnosis; 2 . Angiography was the gold standard for the diagnosis of thrombosis; 3. Ultrasound Doppler could effectively detect vascular patency, which is a powerful screening method. The most common manifestations were the loss of blood flow signal or an increased resistance index $(\mathrm{RI})^{[12]}$. In this study, the early state of thrombosis, such as the slowing and fasting of blood flow, was also regarded as thrombosis. Because according to our previous observations, these abnormal blood flow signals can easily form into thrombosis in the end. In the later analysis process, we classified the patients with abnormal blood flow signal as the thrombosis group. Daily ultrasound examination, which was the most important test, was performed to detect the blood flow within one week after surgery in our center.

\section{Statistical analysis}

All the data were analyzed using the SPSS 23.0 software package for Windows (SPSS, Inc., Chicago, IL, USA). Absolute number (n), frequency, percentage and mean \pm standard deviation (SD) were shown, as appropriate, to describe the data. The $\chi^{2}$ test and Fisher's exact test were used for the categorical variables. Logistic regression models were used to detect the risk factors associated with the Sonoclot 
variables and thrombosis. Data were reported as odds ratios (ORs) with corresponding $95 \%$ confidence intervals (Cls). Receiver operating characteristic (ROC) analysis was used to analyze the effect of Sonoclot parameters on thrombosis. The cutoff value was determined by the maximum Youden index with optimal sensitivity and specificity. Patient survival was analyzed using Kaplan-Meier survival analysis and compared using the log-rank test. A $P$ value $<0.05$ was considered statistically significant for all variables.

\section{Results}

\section{Baseline demographics}

A total of 238 patients underwent regular coagulation monitoring. Among these patients, 5 were retransplant cases, 10 had congenital heart disease or other diseases, 12 had massive hemorrhage during or after surgery, and 11 were lost to follow-up. Finally, 200 patients met the inclusion criteria in this trial.

\section{Postoperative Complications And Outcomes}

A total of 38 cases (19\%) had thrombosis complications in 200 children within 1 week after surgery. Among them, 23 cases (11.5\%) had hepatic artery thrombosis and 15 cases $(7.5 \%)$ had portal vein and hepatic vein thrombosis. 3 patients $(1.5 \%)$ was found thrombosis both in arterial and venous blood vessels. Among patients with abnormal arterial blood flow signals, $12(6 \%)$ had no blood flow signals, $5(2.5 \%)$ showed fast blood flow signals, and 6(3\%) showed slow blood flow signals detected by ultrasound. For patients with abnormal venous blood flow signals, $7(3.5 \%)$ showed no blood flow signals, $4(2 \%)$ showed fast blood flow signals, and $4(2 \%)$ showed slow blood flow signals. Three patients died $(1.5 \%, 2$ patients died of multiple organ failure caused by hepatic artery thrombosis, 1 died of acute rejection) within 1 month after the operation. Four patients (2\%) had to undergo transplantation again because of graft failure. Eight patients underwent balloon dilation to recanalize blood vessels or relieve anastomotic stenosis. The outcomes are shown in Table 1. 
Table 1

Early complications after liver transplantation in children

\begin{tabular}{|llll|}
\hline Complications & Cases(n) & Persentage(\%) \\
\hline Total thrombosis & 38 & 19.0 \\
\hline Hepatic artery thrombosis (HAT) & $\begin{array}{l}\text { No blood } \\
\text { flow }\end{array}$ & 12 & 6.0 \\
\cline { 2 - 3 } & Faster flow & 5 & 2.5 \\
\cline { 2 - 3 } & Slower flow & 6 & 3.0 \\
Portal vein and hepatic vein thrombosis (PVT and & No blood & 7 & 3.5 \\
HVT) & flow & 4 & 2.0 \\
\cline { 2 - 4 } & Faster flow & 4 & 2.0 \\
\cline { 2 - 4 } & Slower flow & 4 & 1.5 \\
\hline Both arterial venous thrombosis & 3 & 1.5 \\
\hline Death & & 3 & 4.5 \\
\hline Secondary into ICU & & 9 & 2.0 \\
\hline Re-transplantation & & 4 & 4.0 \\
\hline Exploratory laparotomy & & 8 & 4.0 \\
\hline Balloon dilatation & & 8 & \\
\hline
\end{tabular}

According to the complications regarding the blood flow signals, we divided the patients into thrombosis group $(n=38)$ and non-thrombosis group $(n=162)$. Two patients in thrombosis group died within 1 month after surgery, and one patient in non-thrombosis group died. Three patients in thrombosis group underwent retransplantation, while one patient in non-thrombosis group underwent retransplantation. The prognosis showed that the early mortality, retransplantation rate, secondary ICU rate, and postoperative laparotomy rate were higher and the ICU stay after surgery was longer in thrombosis group. In addition, the highest level of transaminase (ALT, AST) in thrombosis group was higher than that in non-thrombosis group (Table 2). 
Table 2

Comparison of postoperative conditions between two groups

\begin{tabular}{|lll|}
\hline Outcomes & $\begin{array}{l}\text { Thrombosis group }(\mathrm{n}= \\
\mathbf{3 8})\end{array}$ & $\begin{array}{l}\text { Non-thrombosis group }(\mathbf{n}= \\
\mathbf{1 6 2})\end{array}$ \\
\hline Death [n(\%)] & $2(5.3 \%)$ & $1(0.6 \%)^{*}$ \\
\hline Re-transplantation [n(\%)] & $3(7.9 \%)$ & $1(0.61 \%)^{*}$ \\
\hline ICU stay time (d) & $4.2 \pm 1.0$ & $3.2 \pm 0.5^{*}$ \\
\hline Secondary into ICU [n(\%)] & $4(10.5 \%)$ & $5(3 \%)^{*}$ \\
\hline Post-laparotomy [n(\%)] & $5(13.1 \%)$ & $3(1.9 \%)^{*}$ \\
\hline $\begin{array}{l}\text { Postoperative ALT peak, IU/L } \\
\text { [median(IQR)] }\end{array}$ & $458.4(178.9-498.3)$ & $287.4(198.9-328.2)^{*}$ \\
\hline $\begin{array}{l}\text { Postoperative AST peak, IU/L } \\
\text { [median(IQR)] }\end{array}$ & $145.5(80.8-275.4)$ & $78.3(65.9-154.8)^{*}$ \\
\hline Note: ${ }^{*} P<0.05$. & & \\
\hline
\end{tabular}

We compared the three parameters of the Sonoclot monitor at $1 \mathrm{~h}$ after reperfusion and found that there was a difference in ACT between the two groups, and there were no significant differences in CR or PF between the two groups. The ACT value was lower in thrombosis group than in non-thrombosis group $(178.5 \pm 33.2 \& 280.4 \pm 58.6, P<0.05)$ (Table 3).

Table 3

Comparison of Sonoclot parameters at $1 \mathrm{~h}$ after reperfusion

\begin{tabular}{|lll|}
\hline parameters & Thrombosis group $(\mathbf{n}=\mathbf{3 8})$ & Non-thrombosis group $(\mathbf{n}=162)$ \\
\hline ACT $(\mathrm{s})$ & $178.5 \pm 33.2$ & $280.4 \pm 58.6^{*}$ \\
\hline $\mathrm{CR}$ & $12.4 \pm 5.4$ & $11.5 \pm 5.7$ \\
\hline PF & $3.0 \pm 1.4$ & $2.6 \pm 1.1$ \\
\hline Note: ${ }^{*} P<0.05$. & \\
\hline
\end{tabular}

\section{Predisposing factors for thrombosis within 1 week after liver transplantation}

The results comparing baseline characteristics and perioperative information between the two groups of patients are shown in Table 4. Patients in thrombosis group had a younger age, lower body weight, and higher PELD score. During the surgery, patients in thrombosis group had a longer anhepatic time and graft cold ischemia time. Patients in thrombosis group received more plasma $(P<0.05)$. The other variables were not statistically significant between the two groups (Table 4). 
Table 4

Comparison of preoperative and intraoperative parameters between the two groups

\begin{tabular}{|c|c|c|}
\hline Variables & $\begin{array}{l}\text { Thrombosis group }(\mathrm{n}= \\
\text { 38) }\end{array}$ & $\begin{array}{l}\text { Non-thrombosis group }(n= \\
162)\end{array}$ \\
\hline Age $(m)$ & $6.2 \pm 1.7$ & $7.6 \pm 2.0^{*}$ \\
\hline Male $[n(\%)]$ & $22(57.9)$ & $88(54.3)$ \\
\hline Weight(kg) & $6.9 \pm 1.4$ & $7.5 \pm 2.1$ \\
\hline Height(cm) & $50.7 \pm 11.7$ & $61.6 \pm 7.3^{*}$ \\
\hline Anhepatic time(min) & $54.5 \pm 12.4$ & $42.9 \pm 99.7^{*}$ \\
\hline Pre-AST(IU/L) & $164.7 \pm 34.5$ & $176.4 \pm 42.5$ \\
\hline Pre-ALT(IU/L) & $309.3 \pm 56.2$ & $323.0 \pm 45.8$ \\
\hline $\begin{array}{l}\text { Pretransplant creatinine }(\mu \mathrm{mol} / \mathrm{L}) \text {, } \\
\text { [median(IQR)] }\end{array}$ & $8.4(5.2-16.9)$ & $11.3(5.0-15.6)$ \\
\hline Pre-INR & $1.4 \pm 0.3$ & $1.7 \pm 0.3$ \\
\hline PELD score & $21.1 \pm 3.2$ & $16.2 \pm 2.4^{\star}$ \\
\hline Graft cold ischemia time(min) & $95.4 \pm 16.4$ & $77.4 \pm 15.1^{*}$ \\
\hline $\begin{array}{l}\text { Intraoperative blood transfusion, u } \\
\text { [median(IQR)]) }\end{array}$ & $3.5(1.5-3.5)$ & $3.5(1.0-3.0)$ \\
\hline $\begin{array}{l}\text { Intraoperative frozen plasma } \\
\text { transfusion }(\mathrm{ml})\end{array}$ & $443.5 \pm 126.6$ & $334.5 \pm 174.4^{\star}$ \\
\hline $\begin{array}{l}\text { Intraoperative bleeding volume, ml } \\
\text { [median(IQR)] }\end{array}$ & $200.0(150.0-350.0)$ & $250.0(200.0-350.0)$ \\
\hline Intraoperative urinary volume (ml) & $484.3 \pm 176.5$ & $503.6 \pm 170.2$ \\
\hline Operation time, hours[median(IQR)] & $8.5(7.0-11.0)$ & $8.5(7.5-11.0)$ \\
\hline
\end{tabular}

NOTE: All $P$ values for the variables presented as $\mathrm{n}(\%)$ were obtained with the chi-square test. The variables presented as mean \pm SD were obtained with the Student t test, and the $P$ values presented as median and interquartile range (IQR) were obtained with the Wilcoxon rank sum test. ${ }^{*} P \otimes 0.05$.

We included the meaningful variables in Table 4 and ACT into a multivariate regression analysis and found that PELD [OR $=0.557(1.205-2.529), P=0.003]$, graft cold ischemia time [OR $=0.070(1.002-$ $1.148), P=0.045]$ and $A C T$ [OR $=-0.078(0.884-0.967), P=0.001]$ were independent risk factors for postoperative thrombosis (Table 5 ). 
Table 5

Predisposing factors for postoperative thrombosis by multivariate regression analysis

\begin{tabular}{|llllllll|}
\hline Variables & B & SE & Wald & $P$ & $\operatorname{Exp}(\mathrm{B})$ & $95 \% \mathrm{Cl}$ & \\
\hline PELD & 0.557 & 0.189 & 8.668 & 0.003 & 1.745 & 1.205 & 2.529 \\
\hline Graft cold ischemia time & 0.070 & 0.035 & 4.021 & 0.045 & 1.072 & 1.002 & 1.148 \\
\hline ACT & -0.078 & 0.023 & 11.905 & 0.001 & 0.925 & 0.884 & 0.967 \\
\hline
\end{tabular}

\section{Cutoff Point For Act By Roc Analysis}

ROC analysis was used to identify potential cutoff points for ACT. As shown in Fig. 1 and Table 6, intraoperative ACT ( $1 \mathrm{~h}$ after ischemia-reperfusion) of $>228.5 \mathrm{~s}$ was associated with the maximum Youden index, with optimal sensitivity and specificity.

Table 6

Sensitivity and specificity for the optimal cut-off values of ACT for thrombosis by ROC analysis

\begin{tabular}{|lllll|}
\hline AUC & Sensitivity(\%) & Specificity(\%) & $\mathbf{9 5 \%} \mathrm{Cl}$ & Youden Index \\
\hline 0.92 & 0.947 & 0.802 & $0.934-0.983$ & 228.5 \\
\hline
\end{tabular}

\section{Survival Rate Within 1 Month After Surgery}

The survival rate within 1 month after surgery was found to be lower in the thrombosis group than in the non-thrombosis group (94.7 \& 99.4, $P=0.03$ ) (Fig. 2).

\section{Discussion}

Pediatric liver transplantation is an important part of clinical liver transplantation for children suffering from end-stage liver disease ${ }^{[13]}$. Recently, data promulgated by a Japanese research team showed that the graft survival rates were $90.5 \%, 90.4 \%, 84.6 \%, 82.0 \%, 79.9 \% 1,5,10$, and 20 years after surgery, respectively ${ }^{[14]}$. It was the largest cohort study and followed a total of 2085 children worldwide to observe the overall survival in children with biliary atresia after liver transplantation. The results showed that the postoperative survival rate had improved substantially. However, there are still a series of complications after surgery that affect quality of life. Bleeding and coagulopathy are critical issues complicating pediatric liver transplantation and contributing to morbidity and mortality in cirrhotic children. Vascular complications are common in pediatric liver transplantation ${ }^{[15]}$. In fact, thrombosis remains the primary cause of early graft failure and retransplantation within the first $30 \mathrm{~d}$ after surgery, and it occurs despite prolongation of standard coagulation assays ${ }^{[4]}$. Vessel anastomosis between the donor and recipient is more difficult because the blood vessels do not match well and the blood vessels of children are thinner 
than adults ${ }^{[16,17]}$. The blood flow signal at the anastomosis of the liver vessel tends to be abnormal after operation, which may cause serious complications such as thrombosis. Perioperative coagulation should not be overcorrected; otherwise, thrombosis can easily develop during and after surgery. Thus, it is important to correct coagulation function accurately. This study found that perioperative coagulation affected the occurrence of postoperative liver blood flow signals. The most common vascular complication after surgery was thrombosis. Sonoclot analyzers can be used to monitor coagulation function in pediatric liver transplantation. After ischemia-reperfusion, the Sonoclot parameter ACT was a risk factor for postoperative thrombosis. It was recommended that ACT be maintained above $228.5 \mathrm{~s} 1 \mathrm{~h}$ after ischemia-reperfusion until the end of surgery to reduce the incidence of abnormal postoperative blood flow.

The liver is a site of synthesis for most coagulation factors and anticoagulant substances. The special pathophysiological changes in liver transplantation lead to a large change in perioperative coagulation function. Children with biliary atresia develop cirrhosis before surgery, affecting the synthesis and release of coagulation factors. Clotting factor production is significantly reduced in the perioperative period anhepatic phase. The release of toxic substances that accumulate from the lower part of the body and perfuse the donor liver after ischemia-reperfusion causes a large blow to the neohepatic stage liver ${ }^{[10,18]}$. Liver coagulation function drops again. The coagulation function of the body in the neohepatic stage is the worst. We call this phenomenon the "heparin-like effect" ${ }^{\text {"19] }}$. According to clinical practice, the donor liver was not exposed to heparin in the related liver transplantation, so the coagulation function tended to be stable from $1 \mathrm{~h}$ after reperfusion to the end of the operation. During the anhepatic phase and after ischemia-reperfusion, it is necessary to actively and accurately correct blood coagulation to prevent surgical bleeding and excessive correction of coagulation, which can lead to blood stasis and thrombosis ${ }^{[20]}$.

In fact, thrombosis remains the primary cause of early graft failure and retransplantation within the first $30 \mathrm{~d}$ following surgery, and it occurs despite prolongation of standard coagulation assays. Hepatic artery thrombosis is the most common vascular complication after liver transplantation, with an incidence of $6 \%-15 \%$, of which $22.7 \%$ require secondary liver transplantation ${ }^{[21,22]}$. The portal vein thrombosis rate is $4 \%-10 \%$, easily leading to portal hypertension ${ }^{[4]}$. In our study, the incidence rate of patients with thrombosis after surgery was $19 \%$. Among them, $11.5 \%$ had abnormal arterial signals and $7.5 \%$ had abnormal venous signals. We regarded the patients with abnormal blood flow signals including fast or slow blood flow in the anastomosis or whole blood vessels as thrombosis group. Because patients with these abnormal blood flow signals were likely to eventually form irreversible thrombosis. There are many reasons for the incidence of thrombosis, among which anastomotic stenosis and hypercoagulability are important factors ${ }^{[23]}$. Thus, hypercoagulability during liver transplantation deserves a closer look as thromboembolic events are associated with high morbidity and mortality rates ${ }^{[24]}$.

There are many methods for monitoring coagulation function, including traditional detection of PT, INR, APTT, etc. Some equipment, such as TEG (thromboelastography) and Sonoclot coagulation analyzers 
have been widely used clinically, especially in heart surgery and transplant surgery ${ }^{[25-27]}$. However, the value of these traditional parameters, such as PT and APTT, has been questioned in the acute perioperative setting because of the delay between blood sampling and obtaining the results. Additionally, these tests are carried out on plasma rather than whole blood, and they do not provide much information about platelet function ${ }^{[28]}$. Currently, TEG is widely used in major operations, such as heart surgery and adult liver transplant, because it contains many parameters for accurately monitoring the coagulation system. However, TEG requires specialized personnel and has a high cost. The most important thing is that both traditional detection methods and TEG detection take a long time to obtain results and cannot provide monitoring in real time ${ }^{[29]}$. The environmental changes during the perioperative period of liver transplantation are faster. Therefore, real-time monitoring is required for proper coagulation adjustment. The Sonoclot analyzer is a point-of-care coagulation monitoring system that can immediately observe the patient's coagulation function, such as clotting factor levels, Fib levels, and PF changes. The advantages Sonoclot devices show are that the blood is analyzed at the bedside, allowing a faster turnaround time, and the coagulation status is assessed on whole blood, which is more meaningful than a single index and is an effective means of detecting platelet function ${ }^{[30]}$. Sonoclot devices are used in situations of abnormal coagulation, such as cardiac and liver transplant surgery ${ }^{[8]}$. Sonoclot analyzers monitor three basic parameters: ACT (the reference value is 119-195 s, and the clinical reference significance is greater), $C R$ (the reference value is 7-23) and PF (the reference value is $1.5-4.5)^{[30]}$. Our study proved that Sonoclot devices could be used to guide the monitoring of coagulation function during the perioperative liver transplantation period in children. The most meaningful parameter was ACT. The value of ACT was lower in the thrombosis group, but there were no significant differences in $\mathrm{CR}$ and PF between the two groups. These results were consistent with clinical findings. Generally, we infuse fresh frozen plasma with no infusion of fibrinogen, platelets, etc.

It is very necessary to maintain a state of lower coagulation than normal for pediatric related-living donor liver transplantation due to special physiological and pathological changes and anatomical features. Therefore, the appropriate coagulation parameters according to Sonoclot devices have always been the focus of our research. Under normal circumstances, the Sonoclot analyzer shows that the ACT is 115$195 \mathrm{~s}$. However, in our study, the ACT was maintained at a higher range to reduce postoperative thrombosis. In this experiment, the ROC curve was used to analyze the ACT cutoff value and found that it was better to maintain the ACT above $228.5 \mathrm{~s}$ to reduce the occurrence of abnormal blood flow signals. This cutoff value exceeded the standard ACT value of the Sonoclot machine (119-195 s), and the area under the curve (AUC) was higher than 0.9. The diagnostic effect and reliability were higher. However, we found that the number of postoperative bleeding cases was small. There was no relationship between the intraoperative Sonoclot parameters and postoperative bleeding complications. Therefore, we did not explore the maximum ACT that caused bleeding complications after surgery. In our center, we maintain the ACT below 300 empirically, and no related intraoperative and postoperative excessive bleeding occurs. Of course, this needs further exploration. 
Undeniably, our study had several limitations. First, our study was based on data from a single center. In the future, multicenter research should be performed to verify the accuracy of the ACT value. In addition, we only analyzed the range of Sonoclot parameters that affected postoperative thrombosis, while the cutoff value for Sonoclot parameters that affect postoperative bleeding were not studied.

\section{Conclusion}

In summary, this study progressively proved the factors associated with abnormal early blood flow signals in children after liver transplantation and found that the ACT after ischemia-reperfusion was related to postoperative thrombosis. Additionally, the ACT should be maintained above the normal range to reduce early postoperative thrombosis.

\section{Declarations}

Ethics approval and consent to participate: This study has been approved by the Ethics Committee of Tianjin First Center Hospital in China (Approval Number: 2016N0039KY), and written informed consent was obtained from eligible guardians. We have registered in clinical trial (ClinicalTrials.gov ID: ChiCTR1900026125).

Trial registration: ChiCTR1900026125. Registered 22 September 2019. http://www.chictr.org.cn/showproj.aspx?proj=43481

Consent for publication: Yes.

Availability of data and materials: Not applicable.

Competing interests: No benefits in any form have been received or will be received

from a commercial party related directly or indirectly to the subject of this article.

Funding $₫$ This work was financially supported by the Natural Science Foundation of Tianjin City in China (protocol no. 18JCYBJC27500), Hospital-level project of Tianjin First Central Hospital (protocol no. 2019CF31).

Contributors: Ying Sun designed the study, wrote the protocol, collected the data, and drafted the paper. Min Zhu analyzed the data and assisted in the writing of the manuscript. Feng Liu provided opinions on pediatric research. Lili Jia and Mingwei Sheng gave methodological advice. Sudeshnna Thapa collected the data. All authors contributed to the design and interpretation of the study and to further drafts. Wenli Yu was the guarantor and provided financial support.

Acknowledgements: We would like to thank Sienco (Sienco, Inc., Arvada, CO, USA) for providing us with this machine for our use and research. Thanks to Sudeshnna Thapa for her help in collecting data. 


\section{References}

1. Sundaram SS, Mack CL, Feldman AG, et al. Biliary atresia: Indications and timing of liver transplantation and optimization of pretransplant care. Liver Transpl. 2017;23(1):96-109.

2. Yazigi NA. Long term outcomes after pediatric liver transplantation. Pediatr Gastroenterol Hepatol Nutr. 2013;16(4):207-18.

3. Lee JY, Lim LT, Quak SH, et al. Cholangitis in children with biliary atresia: health-care resource utilisation. J Paediatr Child Health. 2014;50(3):196-201.

4. Piardi T, Lhuaire $M$, Bruno 0 , et al. Vascular complications following liver transplantation: A literature review of advances in 2015. World J Hepatol. 2016;8(1):36-57.

5. Mourad MM, Liossis C, Gunson BK, et al. Etiology and management of hepatic artery thrombosis after adult liver transplantation. Liver Transpl. 2014;20(6):713-23.

6. Hett DA, Walker D, Pilkington SN, et al. Sonoclot analysis. Br J Anaesth. 1995;75:771-6.

7. Dzemali O, Ganter MT, Zientara A, et al. Evaluation of a New Sonoclot Device for Heparin Management in Cardiac Surgery. Clin Appl Thromb Hemost. 2017;23(1):20-6.

8. Chaudhary V, Chauhan S, Choudhury M, et al. Sonoclot analysis in children with congenital heart disease. Asian Cardiovasc Thorac Ann. 2012;20(5):544-7.

9. Nacoti M, Corbella D, Fazzi F, et al. Coagulopathy and transfusion therapy in pediatric liver transplantation. World J Gastroenterol. 2016;22(6):2005-23.

10. Jia Y, Yong-Feng L, Ying C. Coagulation function analysis in perioperative stage of orthotopic liver transplantation an analysis of 95 cases. WCJD. 2009;17(14):1474-6.

11. Nikeghbalian S, Kazemi K, Davari HR, et al. Early hepatic artery thrombosis after liver transplantation: diagnosis and treatment. Transplant Proc. 2007;39(4):1195-1196.

12. Lu Q, Zhong XF, Huang ZX, et al. Role of contrast-enhanced ultrasound in decision support for diagnosis and treatment of hepatic artery thrombosis after liver transplantation. Eur $\mathrm{J}$ Radiol. 2012;81(3):e338-43.

13. Otte JB. Pediatric liver transplantation: Personal perspectives on historical achievements and future challenges. Liver Transpl. 2016;22(9):1284-94.

14. O'Leary JG, Cai J, Freeman R, et al. Proposed Diagnostic Criteria for Chronic Antibody-Mediated Rejection in Liver Allografts. Am J Transplant. 2016;16(2):603-14.

15. Orlandini $\mathrm{M}$, Feier $\mathrm{FH}$, Jaeger $\mathrm{B}$, et al. Frequency of and factors associated with vascular complications after pediatric liver transplantation. J Pediatr (Rio J). 2014;90(2):169-75.

16. Bonnet S, Sauvanet A, Bruno O, et al. Long-term survival after portal vein arterialization for portal vein thrombosis in orthotopic liver transplantation. Gastroenterol Clin Biol. 2010;34(1):23-8.

17. Schwope RB, Margolis DJ, Raman SS, et al. Portal vein aneurysms: a case series with literature review. J Radiol Case Rep. 2010;4(6):28-38. 
18. Warrillow S, Fisher C, Tibballs $\mathrm{H}$, et al. Coagulation abnormalities, bleeding, thrombosis, and management of patients with acute liver failure in Australia and New Zealand. J Gastroen Hepatol. 2019.

19. Agarwal S, Senzolo M, Melikian C, et al. The prevalence of a heparin-like effect shown on the thromboelastograph in patients undergoing liver transplantation. Liver Transpl. 2008;14(6):855-60.

20. Broniszczak D, Szymczak M, Kaminski A, et al. Vascular complications after pediatric liver transplantation from the living donors. Transplant Proc. 2006;38(5):1456-1458.

21. Ackermann O, Branchereau S, Franchi-Abella S, et al. The long-term outcome of hepatic artery thrombosis after liver transplantation in children: role of urgent revascularization. Am J Transplant. 2012;12(6):1496-503.

22. Fujiki M, Hashimoto K, Palaios E, et al. Probability, management, and long-term outcomes of biliary complications after hepatic artery thrombosis in liver transplant recipients. Surgery. 2017;162(5):1101-11.

23. Kaido T, Uemoto S. Does living donation have advantages over deceased donation in liver transplantation? J Gastroenterol Hepatol. 2010;25(10):1598-603.

24. Xia VW, Ho JK, Nourmand H, et al. Incidental intracardiac thromboemboli during liver transplantation: incidence, risk factors, and management. Liver Transpl. 2010;16(12):1421-7.

25. Kovalic AJ, Khan MA, Malaver D, et al. Thromboelastography versus standard coagulation testing in the assessment and reversal of coagulopathy among cirrhotics: a systematic review and metaanalysis. Eur J Gastroenterol Hepatol. 2020;32(3):291-302.

26. Curry NS, Davenport R, Pavord S, et al. The use of viscoelastic haemostatic assays in the management of major bleeding. British Journal of Haematology. 2018;182(6):789-806.

27. Concha PM, Mertz KV, Munoz CG, et al. [Thromboelastography and classical coagulation tests in the preoperative period of liver transplantation]. Rev Med Chil. 2018;146(3):277-81.

28. Rajkumar V, Kumar B, Dutta V, et al. Utility of Sonoclot in Prediction of Postoperative Bleeding in Pediatric Patients Undergoing Cardiac Surgery for Congenital Cyanotic Heart Disease: A Prospective Observational Study. J Cardiothorac Vasc Anesth. 2017;31(3):901-8.

29. Monagle $\mathrm{P}$, Ignjatovic V, Savoia H. Hemostasis in neonates and children: pitfalls and dilemmas. Blood Rev. 2010;24(2):63-8.

30. Whiting P, Al M, Westwood M, et al. Viscoelastic point-of-care testing to assist with the diagnosis, management and monitoring of haemostasis: a systematic review and cost-effectiveness analysis. Health Technol Assess. 2015;19(58):1-228, v-vi.

\section{Figures}




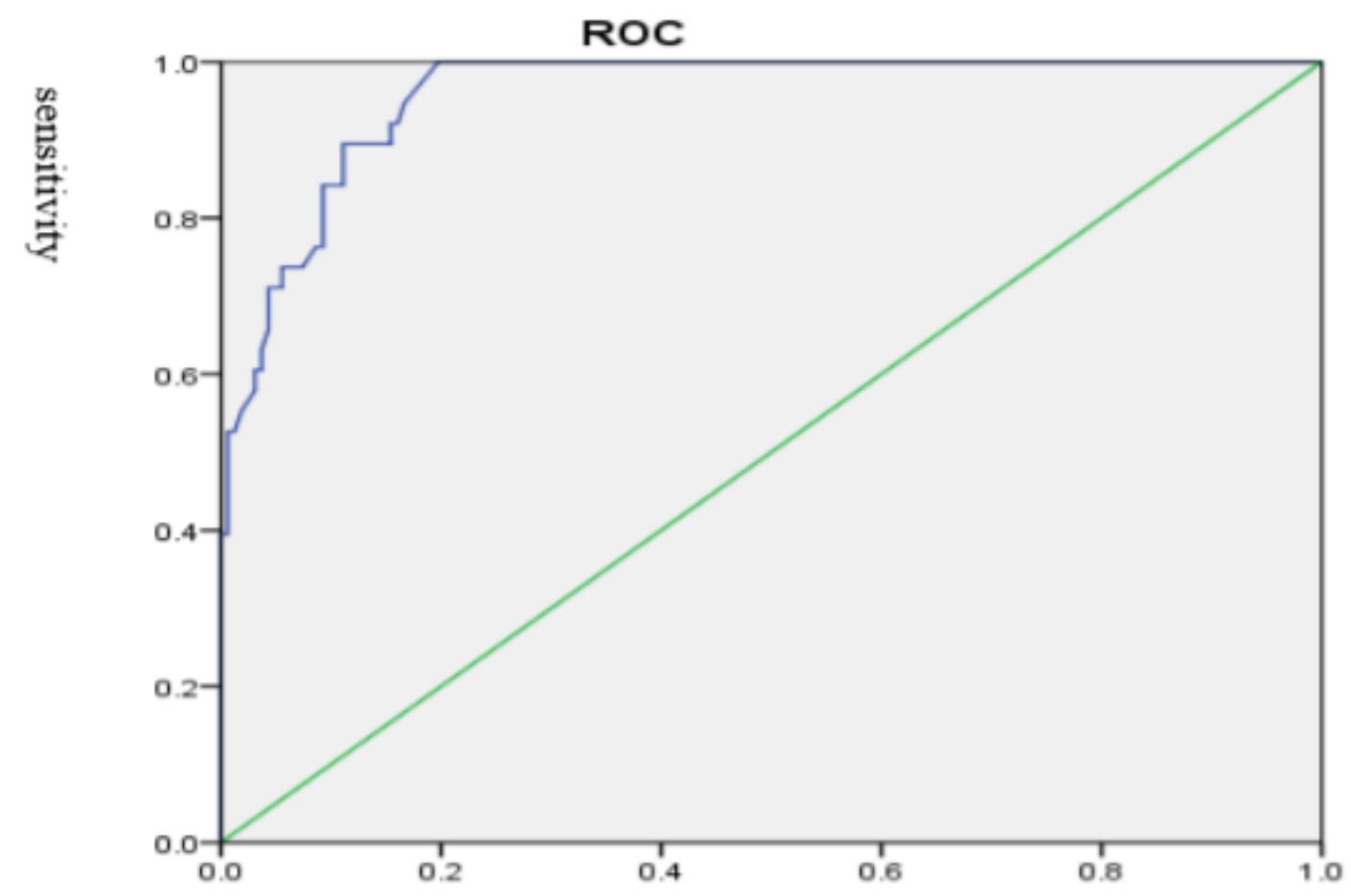

1- specificity

Figure 1

ROC curve analysis of ACT at 1 hour after reperfusion

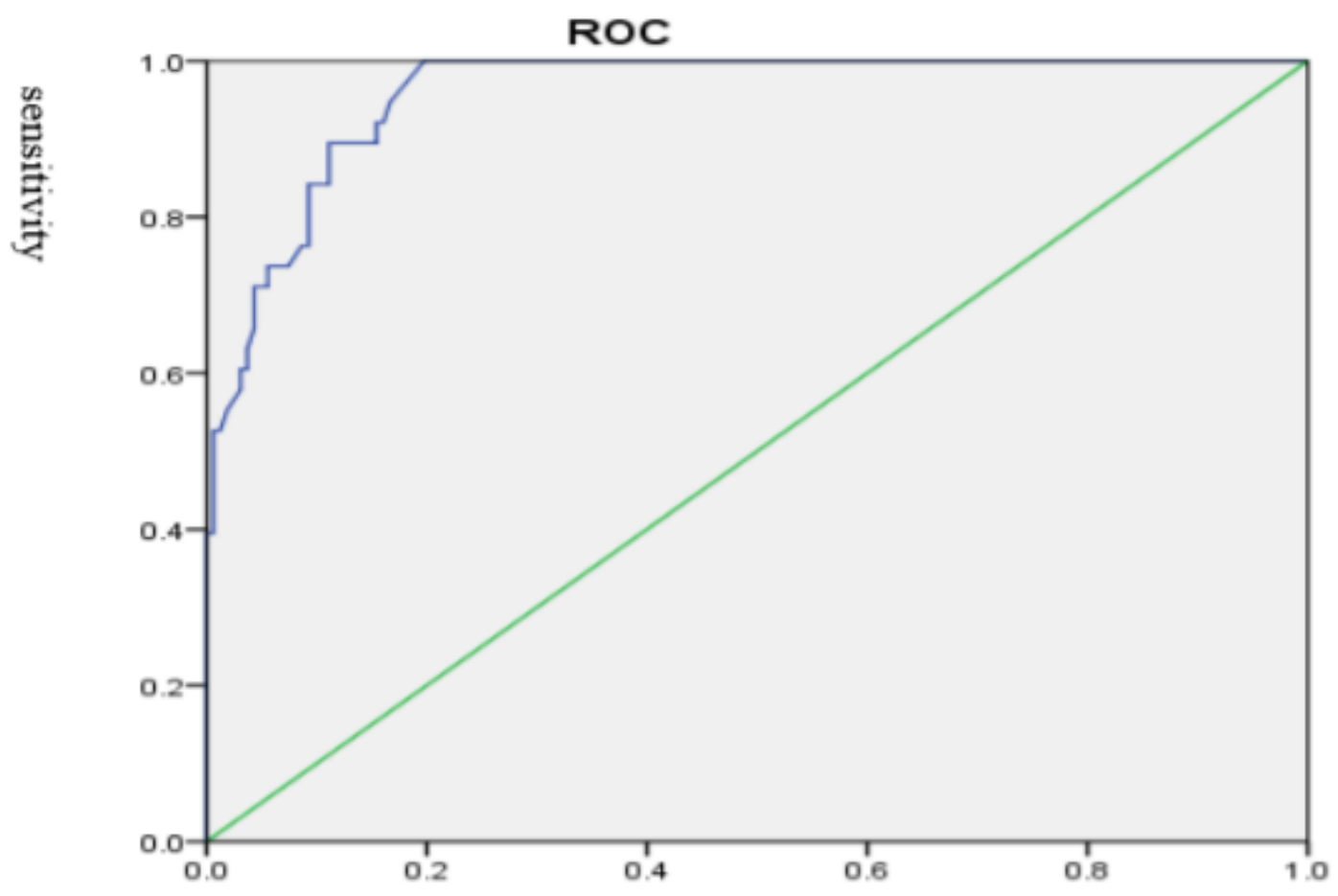

1- specificity

Figure 1 
ROC curve analysis of ACT at 1 hour after reperfusion

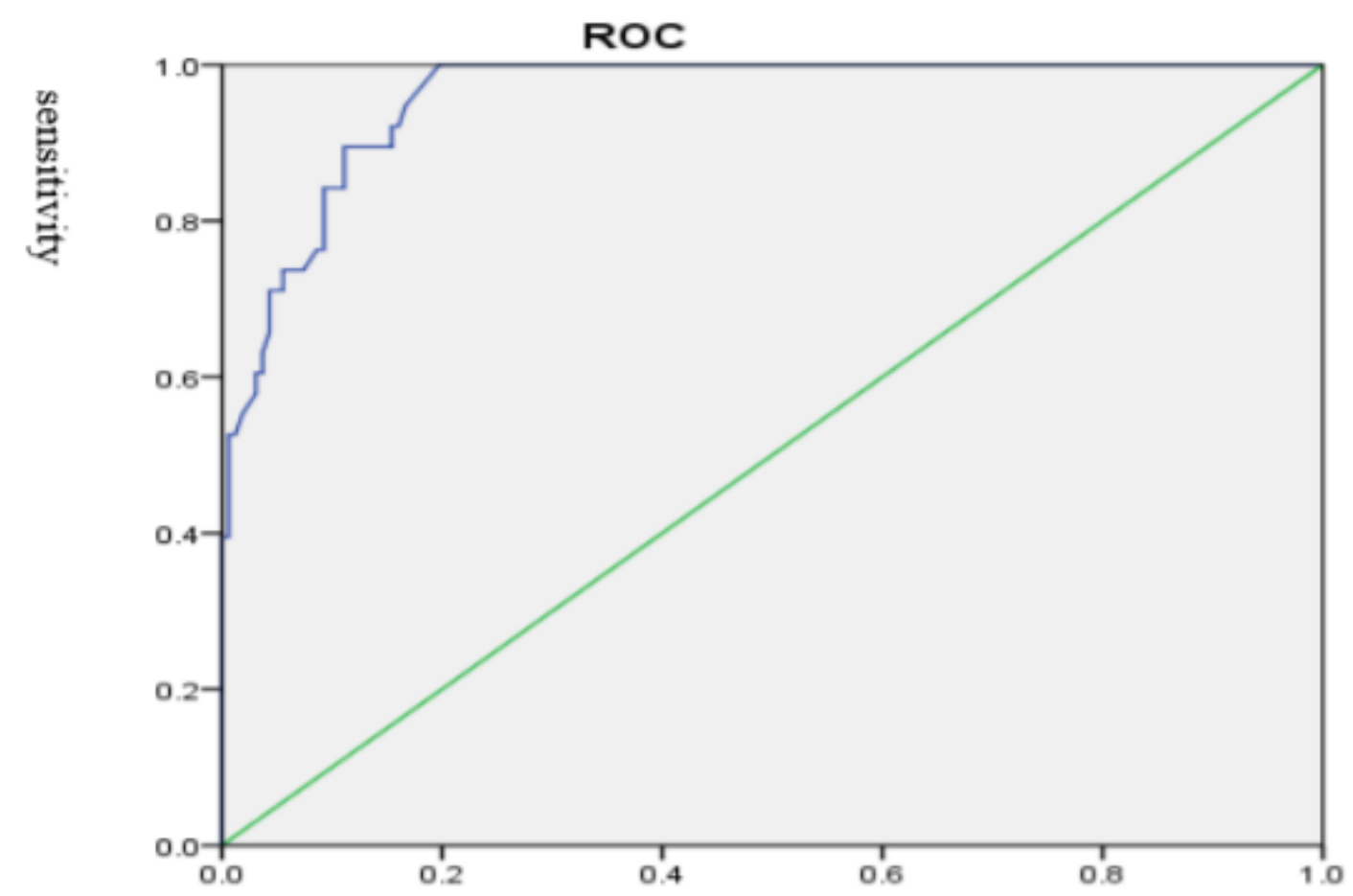

1- specificity

\section{Figure 1}

ROC curve analysis of ACT at 1 hour after reperfusion

\section{Survival proportions}

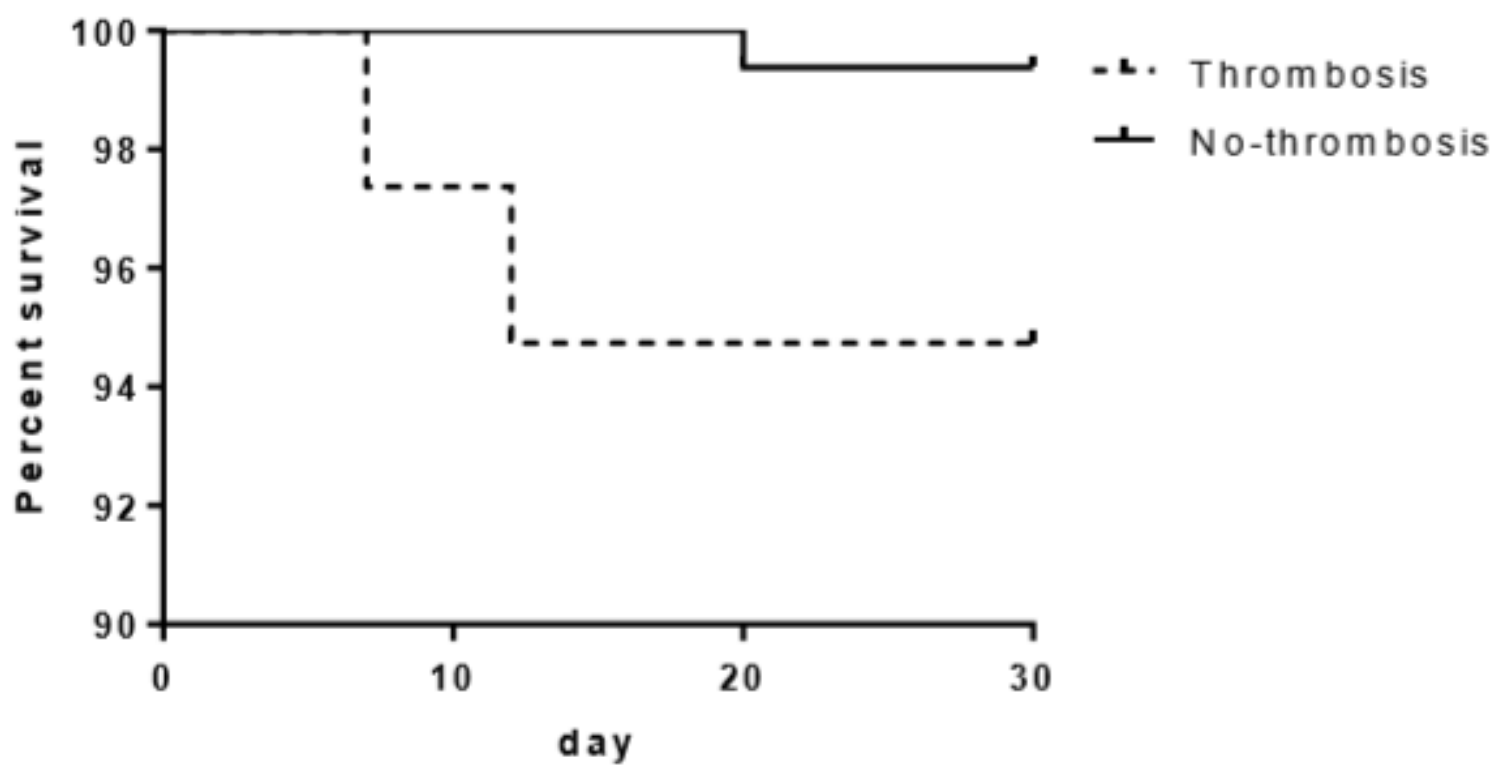

Figure 2

Comparison of survival rate between the two groups 


\section{Survival proportions}

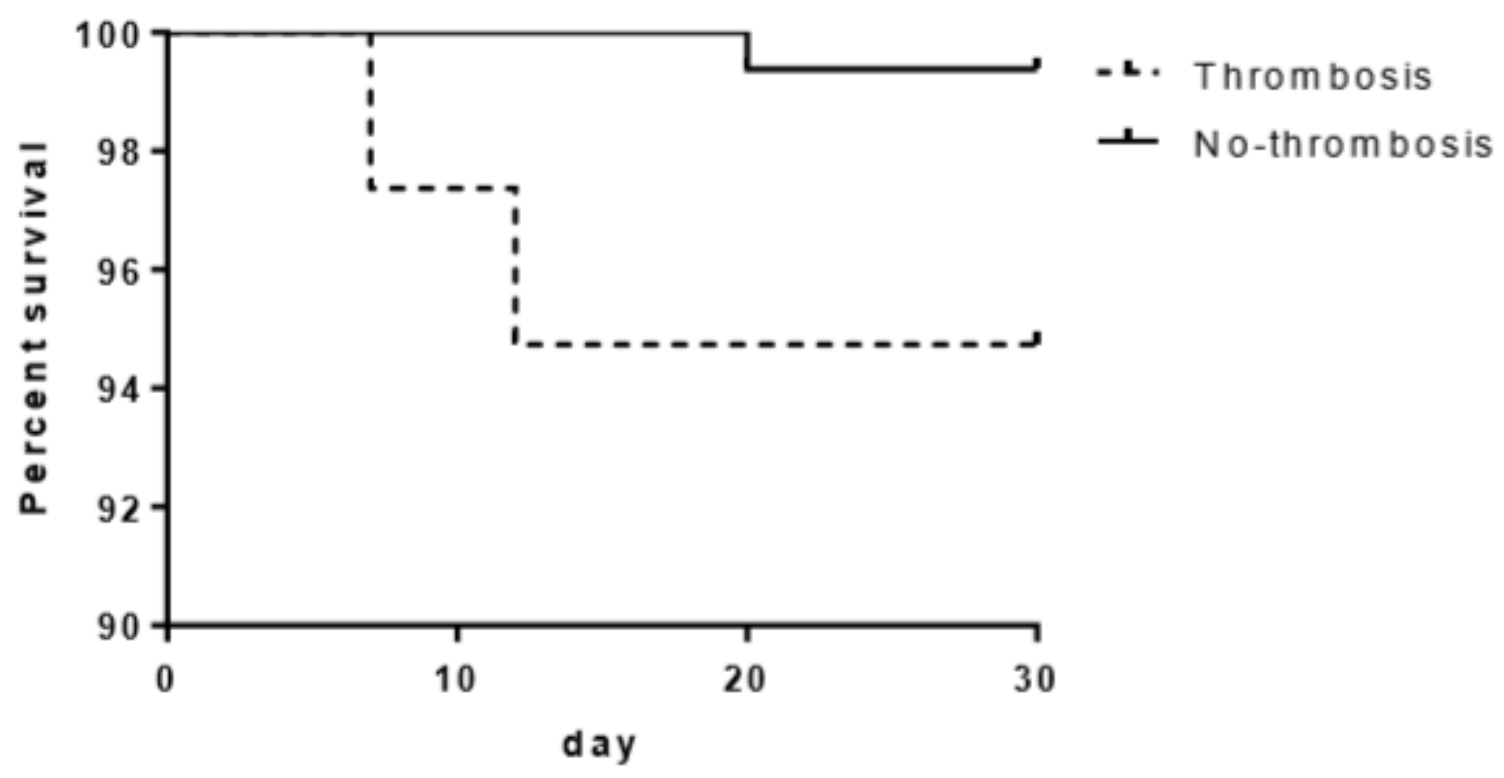

Figure 2

Comparison of survival rate between the two groups

\section{Survival proportions}

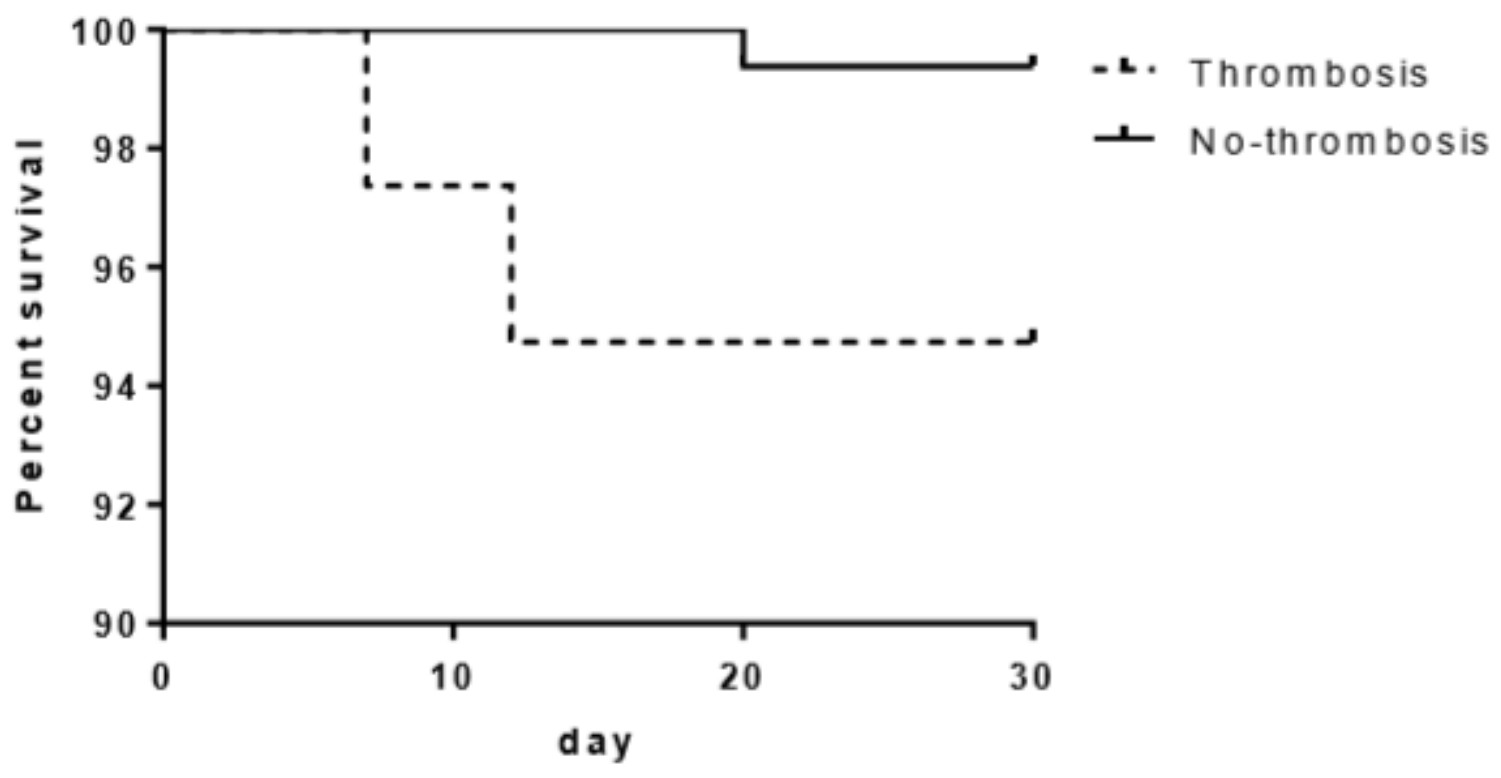

Figure 2

Comparison of survival rate between the two groups 